\title{
Hybrid-Learning-Based Driver Steering Intention Prediction using Neuromuscular Dynamics
}

\author{
Yang Xing, Member, IEEE, Chen Lv, Senior Member, IEEE, Yahui Liu, Yifan Zhao, Senior \\ Member, IEEE, Dongpu Cao, Member, IEEE, Sadahiro Kawahara
}

\begin{abstract}
The emerging automated driving technology poses a new challenge to driver-automation collaboration, which requires a mutual understanding between humans and machines through their intention identifications. In this study, oriented by human-machine mutual understanding, a driver steering intention prediction method is proposed to better understand human driver's expectation during driver-vehicle interaction. The steering intention is predicted based on a novel hybrid-learning-based timeseries model with deep learning networks. Two different driving modes, namely, both hands and single right-hand driving modes, are studied. Different electromyography (EMG) signals from the upper limb muscles are collected and used for the steering intention prediction. The relationship between the neuromuscular dynamics and the steering torque is analyzed first. Then, the hybrid-learningbased model is developed to predict both the continuous and discrete steering intentions. The two intention prediction networks share the same temporal pattern exaction layer, which is built with the Bi-directional Recurrent Neural Network (RNN) and Long short-term memory (LSTM) cells. The model prediction performance is evaluated with a varied history and prediction horizon to exploit the model capability further. The experimental data are collected from 21 participants of varied ages and driving experience. The results show that the proposed method can achieve a prediction accuracy of around $95 \%$ steering under the two driving modes.
\end{abstract}

Index Terms- Automated driving, driver-automation collaboration, steering intention prediction, deep learning, neuromuscular dynamics.

\section{INTRODUCTION}

\section{A. Motivation}

$\mathrm{T}$ HE rapid development of automated driving techniques is posing a series of challenging problems to the automotive industry and academic community. Among these, exploiting the

This work was supported in part by the SUG-NAP Grant (No. M4082268.050) of Nanyang Technological University, the A*STAR Grant (No. 1922500046), the Intra-Create Seed Collaboration Grant Project (NRF2019-ITS005-0011), Singapore, National Natural Science Foundation of China (No.51875302), and the State Key Laboratory of Automotive Safety and Energy Grant (No. KF2021).

(Corresponding authors are Chen Lv and Yahui Liu)

Y. Xing is with the Department of Computer Science, University of Oxford, Oxford, OX1 3QD, U.K. (E-mail:yang.xing@cs.ox.ac.uk).

C. Lv is with the School of Mechanical and Aerospace Engineering, Nanyang Technological University, 639798, Singapore. (E-mail: lyuchen@ntu.edu.sg) roles that a human driver can play in the future automated driving vehicles and how the human and automation can efficiently collaborate is one of the essential tasks [1-3]. Mutual understanding, as a critical aspect for multi-agent teaming and collaboration, enables the human driver and vehicle automation to collaborate efficiently by understanding the capability, intention, and attitudes of the teammate $[4,5]$.

A widely accepted taxonomy for automated vehicles is defined by the Society of Automotive Engineering (SAE). The vehicle automation is divided into six levels, from Level 0 (fully manual control) to Level 5 (fully automated driving) [6]. There is a consensus that automated vehicles are designed to serve humans better in terms of driving safety and transportation efficiency. [7]. On the one hand, autonomous vehicles are advantageous over human drivers in responsiveness, computation, and simultaneous operations. On the other hand, however, human drivers outperform automation with respect to decision-making in complex situations, policy and ethical understanding, and long-term memory [8]. Although vehicle automation can help reduce the cognitive load of a human driver, the coordination of human and automation is also challenging [9]. Human-machine teaming with hybrid intelligence is an emerging research topic, and it is of great importance for realizing the harmonic co-existing of a human driver and vehicle automation [10]. Before realizing fully autonomous driving, driver cognition and human-machine interaction toward better mutual understanding should be well addressed in the system design of automated vehicles.

Hence, in this study, a driver steering intention prediction system is developed for vehicle automation to understand human intention further so that a collaborative automated driving system can be developed in the future.

Y. Liu is with the School of Vehicle and Mobility, Tsinghua University, Beijing 100084, China. (E-mail: liuyahui@tsinghua.edu.cn)

Y. Zhao is with the School of Aerospace, Transport and Manufacturing, Cranfield University, Bedford, MK43 OAL, UK. (E-mail: yifan.zhao@cranfield.ac.uk)

D. Cao is with the Dept. of Mechanical and Mechatronics Engineering, University of Waterloo, ON, N2L 3G1, Canada (E-mail: dongpu.cao@uwaterloo.ca)

S. Kawahara is with the Research and Development Headquarters, JTEKT Corporation, Kashihara, Nara 634-8555, Japan (E-mail: sadahiro kawahara@jtekt.co.jp) 


\section{B. Literature Review}

Driver steering intention plays a critical role in the development of human-centered automated driving systems $[11,12]$. Specifically, two important automated driving techniques can benefit from the integration of driver intention prediction. First, the shared control and shared steering strategy can be optimized by involving the human steering intention $[13,14]$. The continuous prediction of the future steering intention, such as the steering torque, will provide an essential clue to the shared steering control systems. A compensate and optimized steering strategy can be determined by the automation in advance to guarantee driving safety in both typical and critical environments [15]. Second, for the partially automated driving vehicle, the control authority transition between the human driver and the automation should be safe and smooth [16]. It is crucial to evaluate the driving performance of the human driver after a take-over maneuver, as driving skill degradation can be experienced by the human driver after a period of automated driving [17]. Hence, predicting the driver steering intention will allow vehicle automation to assess the future driving behaviors, estimate the driving risks, and provide necessary assistance to the driver.

Existing studies on driver intention prediction mainly focus on the tactical intention inference, such as braking, lane change, and turn intention [18-20]. It is shown that the lane change intention can be predicted up to $3.5 \mathrm{~s}$ before the maneuver in highway and urban road with over $80 \%$ prediction accuracy [21]. Most of the studies focus on the discrete intention classification and prediction based on the integration of video sequences with the inside and outside context, digital maps, GPS, and Lidar information. Complex sensor fusion and data coordination are usually needed. For example, in [22], an ensemble LSTM based model was proposed for highway lane change intention inference based on the camera and vehicle dynamic information. In [23], the driver behavior features, road context, and digital map information are fused for the lane change and turning intention prediction. In [24], a relevance vector machine (RVM) classifier was applied to estimate the lane change intent by integrating a series of Advanced Driver Assistant Systems (ADAS) modules. The multimodal signals from Adaptive Cruise Control (ACC), Side Warning Assistance (SWA), Lane Departure Warning (LDW), and head motion were fused. It was found that the LDW system was more useful to predict the intent between 0 and 1.5 seconds before the lane change occurs. Although the tactical intention can be predicted with a relatively larger prediction horizon (normally $0 \mathrm{~s}$ to 3.5 $\mathrm{s}$ in advance), only the discrete intention states can be estimated due to the lack of connections between human physical dynamics and the steering behaviors.

The neuromuscular dynamics and the EMG singles of the driver have been widely studied in the past decades. Existing studies mainly focus on the steering assistant system design for advanced driver assistant systems (ADAS), haptic shared control, and take-over control [25-27]. Abbink et al. found that an improper mapping between the driver neuromuscular dynamics and the assistant steering torque would significantly decrease the performance of the haptic shared steering control system [28]. Similarly, Liu and Guo et al. showed that a tradeoff between the freedom of driver operation and the safety assurance of the vehicle could be made with a model predictive control (MPC)-based shared control method [29]. In [30], Pick and Cole studied the co-relationship between the EMG signals from the upper body and the steering torque. It was shown that the sternal portion of the pectoral and the mid-portion of the deltoid are the two most important muscles to the generation of the steering force. A multiple regression model was developed for the steering torque estimation. While the correlation analysis and the steering torque regression were studied, the continuous sequential steering torque cannot be precisely estimated with the regression model.

In [31], driver neuromuscular dynamics were studied to explain the different muscle activity during steering. It was shown that the driver tends to perform "push steering" during clockwise and counterclockwise steering. Moreover, the driver steering workload can be estimated based on the measurement of muscle alternation and co-contraction. In [32], an estimation method for steering comfort and efficiency was developed. The effective steering force, as well as the total force capability, can be calculated based on the multi-regression of the steering force and EMG signals. Although the EMG signals and neuromuscular dynamics have been studied for steering behavior modeling and estimation widely, the steering intention prediction with a longer prediction horizon for both continuous and discrete intention prediction is still needed to be exploited and quantitively analyzed. Hence, in this study, a hybridlearning-based steering intention prediction system that considering the EMG signals is designed to benefit both the collaborative decision-making and steering assistant control system for the automated driving vehicle in the future.

\section{Contribution}

The contribution of this study can be summarized as follows. First, the hybrid-learning-based time-series modeling approach enables the continuous steering torque prediction and the discrete intention classification. With the proposed hybridlearning network, both the continuous and discrete prediction can be the critical features and inputs to the automated decisionmaking and collaborative driving algorithms. Second, the neuromuscular dynamics from the upper limb muscles are studied and used for the time-series model training. Based on the cross-correlation analysis between the EMG signals and the steering torque, it is shown that a strong correlation exists between the signals, and the history observation can lead to a precise prediction of the steering intention with a certain prediction horizon. Last, the model performance on the steering intention classification is evaluated based on different history and prediction horizons. The model capabilities in the steering intention prediction are exploited and discussed.

\section{Paper Organization}

The organization of this study is as follows. In section II, a high-level system overview is introduced. In section III, the experiment platform, the testing scenarios, and the data analysis for the EMG and steering torque signals are described. The model structures and training process for the deep time-series model are highlighted in Section IV. In Section V, experiment results are evaluated and compared with baseline methods. Last, conclusions are made in section VI, 


\section{HIGH-LEVEL ARCHITECTURE OF THE SYSTEM}

In this section, the high-level architecture of the proposed system is introduced. The hybrid-learning-based driving intention prediction system contains four separate parts in this study, which are data collection, data processing, model construction, and performance evaluation. The overall system architecture is shown in Fig. 1 below.
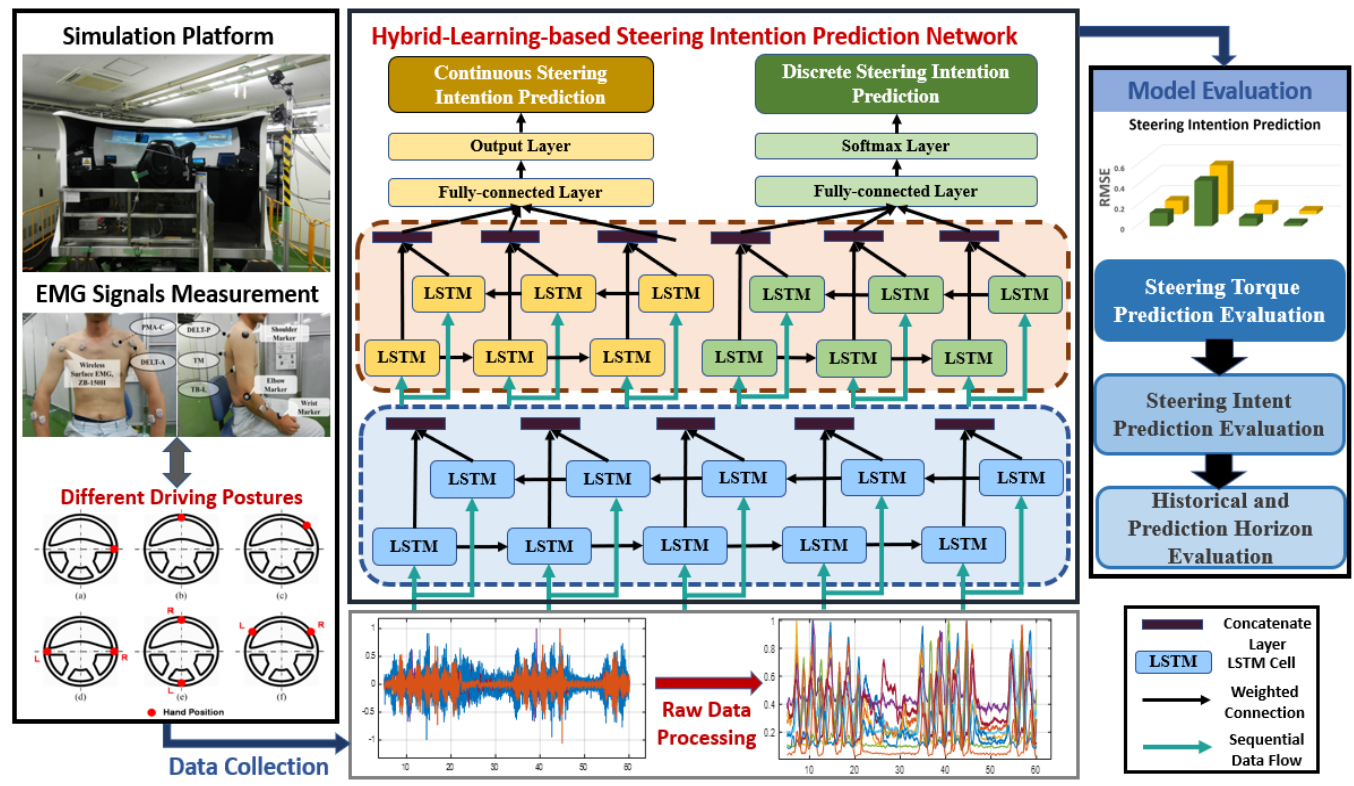

Fig. 1. The high-level system architecture of the proposed driver steering intention prediction system.

First, in the data collection section, the naturalistic steering data and the corresponding EMG signals are collected via a driving simulator. Due to availability reasons, 21 male subjects participated in the EMG data collection experiment. It should be noted that although individual drivers may have different muscle responses under the same driving tasks, the deviation of their neuromuscular dynamics can be controlled with the predefined driving postures and tasks. For recruited participants, their human upper limb dynamics show a similar pattern, and the distribution of the EMG signals does not change significantly. Hence, the dataset that is collected from 21 participants can provide a fair evaluation.

To capture as many steering behaviors and neuromuscular dynamics as possible, each participant will perform two driving modes, namely, both-hand driving mode and single-hand (with right hand on the steering wheel) driving mode. Moreover, for each driving mode, the participants will adopt three driving postures with different hand positions on the steering wheel to ensure sufficient neuromuscular dynamics can be collected. Ten EMG signals from the upper limb are collected for each participant. Detailed information for the collected EMG signals regarding the driving mode is shown in the next section.

In the data processing stage, the EMG signals and the steering torque are filtered with a bandpass filter $(10-500 \mathrm{~Hz})$ and smoothed to remove outliers and noise. The EMG data are normalized, and the absolute version of the signal is used. Then, the cross-correlation analysis for the EMG signals and the steering torque is performed to explore the relationship between the signals. The correlation and time delay metrics are used to evaluate the relationship between the EMG signals and the steering torque. The analysis provides quantitive measurements between the neuromuscular dynamics and steering torque. The time delay measures how the two signals best aligned with each other and will contribute to a clear understanding of the relationship between the neuromuscular dynamics and the steering torque in the time-domain. This part is also discussed in the next section.

The hybrid-learning-based time-series model will be described in Section IV. The time-series model uses deep RNN and LSTM cells to predict the two different steering intentions jointly. The continuous intention prediction model predicts the future steering torque with a pre-defined prediction horizon. And the discrete prediction module identifies the driver's steering intention by five categories (right steering, right steering back, left steering, left steering back, and hold) to support a fast intention estimation. The continuous and discrete intention prediction modules share the same temporal pattern extraction layer, which is trained based on the sequence prediction dataset for the continuous intention prediction. Then, personalized $\mathrm{Bi}$-directional RNN layer and prediction layers are developed for each task to precisely estimate the two types of intention. The continuous sequence prediction of the steering torque will contribute to the precise steering assistant and shared steering control. The discrete steering intention prediction module classifies five steering behaviors according to the variation of the steering torque. The predicted discrete intention can be an essential input to the take-over performance assessment system and a high-level collaborative decisionmaking system for the automated driving vehicle.

Last, model performance on the two intention prediction tasks will be quantitively evaluated and compared with baseline methods. Moreover, for the discrete intention prediction task, the evaluation of the historical horizon and the prediction horizon will be proposed to investigate the impact of these hyperparameters on the model prediction accuracy. The results 
will contribute to the design of the steering intention prediction system, considering the neuromuscular dynamics.

\section{EXPERIMENT DESIGN AND DATA ANALYSIS}

In this section, the experiment platform and experiment design procedure are introduced first. Then, based on the collected EMG and steering torque data, the cross-correlation analysis is proposed to analyze the relationship between the different data.

\section{A. Experiment Design}

In this study, the EMG data are collected from 21 male participants with varied age and driving experience from the driving simulator platform. The experimental platform is a human-in-the-loop driving simulator, as shown in Fig. 1. The CarSim simulation platform is used for the construction of the driving simulation scenarios. A steering system with a hydraulic servo-device is adopted and placed under the cockpit to mimic the dynamic load of the vehicle and provide feedback responses to human participants via the steering system. Each participant is instructed to perform a sine steering like the slalom steering test. The magnitude of the sine steering angle is around $60 \mathrm{deg}$, and the corresponding load provided by the driving simulator is with a magnitude of $5 \mathrm{~N} \cdot \mathrm{m}$ and a frequency of $0.25 \mathrm{~Hz}$. The simulated load is generated based on the driving scenario of the slalom steering test of a passenger car under the velocity of $60 \mathrm{~km} / \mathrm{h}$. Each participant is required to perform the sine steering for at least five circles continually. A force sensor (DynPick WEF-6A1000) and a torque angle sensor (TR-60TC) are mounted under the steering wheel to detect the real-time steering dynamics. The EMG signals are measured by Nihon Kohden $\mathrm{ZB}-150 \mathrm{H}$ wireless sensors with a sampling frequency of $1000 \mathrm{~Hz}$.

The electrodes are all placed in the center of relevant muscles and should be kept as far apart as possible and maintain sufficient distance to prevent interference. In the experiment, 21 participants are all maintained a good health condition without any musculoskeletal anomalies before and during the experiment. All of the participants are deeply informed about the purpose and risks of this experiment and agreed to participate. The participants are aged between 20 and 40 years old. The 21 participants can be roughly divided into three groups based on their driving experience. Specifically, seven drivers have less driving experience, seven drivers have average driving experience, and the rest are seven skilled drivers, respectively. Involving multiple drivers with different driving experience can avoid bias in the pattern analysis of the neuromuscular signals and increase data diversity. During the experiments, the 21 participants are asked to steer with a constant sinusoidal angle input with the two different driving modes, which are driving with both hands and single right arm driving. The test subject must hold the steering wheel fixed with the 3 -clock postures at the beginning.

As shown in Fig. 1, three different hand positions are also investigated for the two driving modes, respectively. For the single hand driving mode, three driving postures, namely, 3clock, 12-clock, and 1-clock, are studied. Similarly, for the both-hand driving mode, three postures, namely, 3-clock, 12clock, and 10-10-clock position, are investigated. The selection of different hand positions is based on the investigation of driving habits, related knowledge, and experience among the experiment designers as well as the participants. All the six hand positions are typical postures during their daily driving activities. The participants are required to perform the maneuver three to five times. Hence, each measurement should have 12-15 periods of torque signals with about five to ten seconds stop after every three continuous periods. An exemplar illustration of the steering task is shown in Fig. 2. The purpose of this task is to validate the quantitative
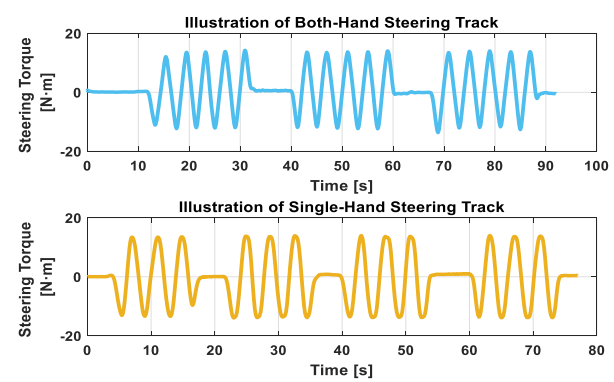

Fig. 2. Exemplar illustration of the steering torque signals with bothhand and the single-hand driving mode, respectively.

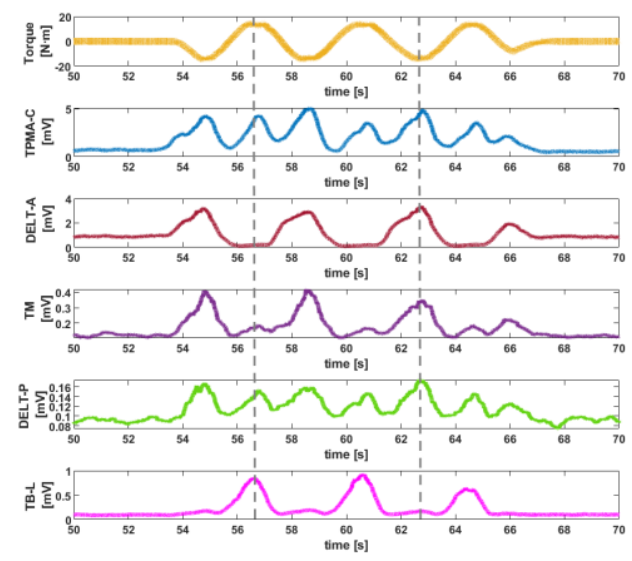

Fig. 3. Sequential steering torque and EMG signals (after raw data processing) from the right upper limb.

method of steering intention by checking the mapping relation between the EMG signals and the steering torque. The study protocol and consent form are approved by the JTEKT Corporation, Japan, and consent is obtained from all subjects.

\section{B. EMG Data Analysis}

For the single-hand and both-hand driving mode, ten electrodes are placed separately in different regions of the upper limb for the EMG signal collection. For the single right-hand driving mode, ten electrodes are attached to the right part of the upper limb, while for the both-hand driving mode, each side of the upper limb has five electrodes. For the both-hand driving mode, ten EMG signals (MB1-MB10) from the right and left upper limb are measured, which are the pectoralis major of clavicular portion, deltoid anterior, deltoid posterior, triceps long head, and teres major of the right and left arms, respectively. Ten different EMG signals (MS1-MS10) from the right upper limb are also measured in the single-hand driving scenario, which are the pectoralis major of clavicular portion, deltoid anterior, deltoid middle (lateral), deltoid posterior, 
triceps long head, triceps lateral head exterior, biceps, infraspinatus, pectoralis major, and teres major. According to [33], the selected five muscles and the corresponding EMG signals can be an efficient clue for the estimation of the steering efficiency. Besides, the overall muscle contribution to the steering maneuver was analyzed in [34]. It shows that the most important muscles to the steering task are the pectoralis major of the clavicular portion and deltoid anterior of both upper limbs. Hence, for the both-hand driving mode, only five electrodes are remained for each side of

TABLE I

CROSS-CORRELATION ANALYSIS BETWEEN THE EMG SIGNALS AND THE STEERING TORQUE

\begin{tabular}{|c|c|c|c|c|c|c|c|c|c|c|}
\hline Both-Hand & MB1 & MB2 & MB3 & MB4 & MB5 & MB6 & MB7 & MB8 & MB9 & MB10 \\
\hline Correlation & 0.7270 & 0.8619 & 0.5877 & 0.5975 & 0.6587 & 0.8274 & 0.8797 & 0.6138 & 0.6101 & 0.6761 \\
\hline Delay (ms) & -192 & -419 & -163 & -49 & -102 & -258 & -326 & -66 & -169 & -157 \\
\hline $\begin{array}{c}\text { Single- } \\
\text { Hand }\end{array}$ & MS1 & MS2 & MS3 & MS4 & MS5 & MS6 & MS7 & MS8 & MS9 & MS10 \\
\hline Correlation & 0.8774 & 0.7670 & 0.7417 & 0.7157 & 0.7995 & 0.7064 & 0.6710 & 0.7071 & 0.8276 & 0.7837 \\
\hline Delay (ms) & -216 & -390 & -202 & -229 & -81 & -177 & -363 & -306 & -52 & -51 \\
\hline
\end{tabular}

the upper limb to measure the most significant neuromuscular dynamics during the steering and save the time cost for the experiment setup.

The visualization of the steering torque and some EMG signals are shown in Fig. 3. As shown in Fig. 3, different muscles have different responses to the steering maneuver. For example, the DELT-A muscle shows significant variation when the driver is performing negative steering torque, while the TB$\mathrm{L}$ muscle is more sensitive to the positive steering torque. It can be found that the activities of the upper limb muscles are closely related to the steering torque and the steering maneuver. Hence, to make a precise prediction of the future steering torque, it is necessary to study the relationship between the EMG signals and the steering torque, which including the correlation and phase shift. In this part, the cross-correlation analysis between the EMG signals and the torque is proposed in the first to gain a visualization between the EMG signals and steering torque. The true cross-correlation $R_{x y}$ of two sequence $x_{n}, y_{n}$ with the length of $n$ can be calculated as follows [35].

$$
R_{x y}(m)=E\left[x_{n+m} y_{n}^{*}\right]
$$

where $n$ is a finite number in the real-world application, $m$ is the displacement, and the asterisk denotes complex conjugation and $E[\cdot]$ is the expected value operation. The cross-correlation is then normalized as follows.

$$
\hat{R}_{x y, \text { coeff }}(m)=\frac{1}{\sqrt{\hat{R}_{x x}(0) \hat{R}_{y y}(0)}} \hat{R}_{x y}(m)
$$

Further, based on the calculated cross-correlation sequence, the relevant time delay can be found at the point where the maximum correlation is achieved, which also indicates where the two sequences are best aligned. The statistics of the correlation and time delay between the EMG signals and the steering torque are represented in Table 1. As shown in Table 1 , most of the measured EMG signals show a medium $(0.5<$ corr $<0.75)$ and strong $(\operatorname{corr} \geq 0.75)$ correlation to the steering torque. For the both-hand driving mode, the MB2, MB6, and MB7 muscles show a significantly strong correlation to the steering maneuver. While, for the single-hand driving mode, the MS1, MS2, MS5, MS9, and MS10 show a strong correlation to the steering torque. Regarding the time delay characteristics, all of the muscles show a negative time delay to the steering torque with the active steering maneuver, which means the variation of the steering torque is determined by the neuromuscular dynamics. The mean value of the time delay between the EMG signals and the steering torque of the ten muscles are $-197 \pm 118 \mathrm{~ms}$ and $-207 \pm 121 \mathrm{~ms}$, for both-hand and single-hand driving modes, respectively. It is shown that the average time delay between the EMG signals and the steering torque is about $200 \mathrm{~ms}$. In the next two sections, the prediction and historical horizon will be chosen according to this time delay analysis, and a series of sliding window with size from 100 to 1000 will be evaluated for steering intent prediction.

\section{HYBRID-LEARNING-BASED TIME-SERIES MODEL}

In this section, the hybrid-learning-based time series modeling approach for the sequential steering torque and discrete steering intention prediction is introduced.

\section{A. Model Construction}

In this study, the hybrid-learning-based model is designed to estimate two different steering intentions, namely, the continuous steering torque and discrete steering intention. The continuous steering torque prediction module, as shown in Fig. 1 , will construct a sequence-to-sequence prediction network to predict the continuous steering torque based on the historical EMG data. Then, by categorizing the steering torque into five states based on its variation, the discrete steering intention can be recognized.

The construction of the hybrid-learning-based intention prediction model contains two stages. First, the continuous steering prediction network can be represented as follows.

$$
S_{t}=f c_{p}\left(f c_{s}\left(\text { bilstm }_{s}\left(\text { bilstm }_{c}\left(\operatorname{In}_{t}\right)\right)\right)\right)
$$

where $f c_{p}$ is the final fully-connected layer for the sequential prediction, $f c_{s}$ is the fully-connected layer with 100 neurons, bilstm $_{s}$ is the personalized Bi-directional LSTM RNN network with 40 LSTM cells in each direction for the sequential steering torque prediction, and bilstm $_{c}$ is the common Bi-directional LSTM RNN network with 60 LSTM cells in each direction for common temporal pattern extraction

The Bi-RNN model can be represented as [36].

$$
\begin{aligned}
& s_{f t}=f\left(\boldsymbol{W}_{1 f} x_{t}+\boldsymbol{W}_{2 f} s_{f t-1}+b_{x}\right) \\
& s_{b t}=f\left(\boldsymbol{W}_{3 f} x_{t}+\boldsymbol{W}_{\mathbf{5} f} s_{b t-1}+b_{x}\right) \\
& o_{t}=f\left(\boldsymbol{W}_{4 f} s_{f t}+\boldsymbol{W}_{6 f} s_{b t}+b_{o}\right)
\end{aligned}
$$

where $f$ is the activation function of the states, which can be tanh and sigmoid function, $X_{t}, S_{f t}, S_{b t}$ and $O_{t}$ are the input, forward hidden states, backward hidden states, and the output of the RNN at the time step, respectively. $\boldsymbol{W}_{1 f}$ and $\boldsymbol{W}_{2 f}$ are the weight matrix for the input and hidden states along the forward direction. $\boldsymbol{W}_{3 f}$ and $\boldsymbol{W}_{5 f}$ are the corresponding weight 
matrix along the backward direction. $\boldsymbol{W}_{4 f}$ and $\boldsymbol{W}_{6 f}$ are the output weight matrix. The output. $b_{x}$ and $b_{o}$ are the input and output bias vector, respectively. A more detailed description of the Bi-directional RNN and LSTM states can be found in [37].

$S_{t}$ in (3) is the predicted sequential steering torque at time $t$, which can be described as:

$$
S_{t}=\left(\hat{s}_{t+1}, \hat{s}_{t+2}, \cdots, \hat{s}_{t+p}\right)
$$

where $\hat{s}_{t+p}$ is the predicted steering torque at time step $t+$ $p, p$ is the prediction horizon, which is selected as 200 as described in the last section. The model input $I n_{-} c_{t}$ can be described as:

$$
\begin{gathered}
\text { In_ } c_{t}=\left\{\left(e m g_{1, t-h}, e m g_{2, t-h}, \cdots, e m g_{10, t-h}, s_{t-h}\right.\right. \\
e m g_{1, t-h+1}, e m g_{2, t-h+1}, \cdots, e m g_{10, t-h+1}, s_{t-h+1}, \\
\left.\left.\cdots, e m g_{1, t}, e m g_{2, t}, \cdots, e m g_{10, t}, s_{t}\right)\right\}
\end{gathered}
$$

where $e m g_{i, t-h}$ is the $i^{\text {th }}$ EMG signal at time step $t-h, h$ is the historical horizon that is used to control the length of the input. In the continuous steering torque prediction case, the historical horizons and the prediction horizon are selected as 200 according to the average time delay. In sum, the dimension of the model input sequence $\left(I n_{-} c\right.$ ) is $11 \times 200$, and the dimension of the output sequence $(S)$ is $1 \times 200$. A meansquared error loss function $\operatorname{loss}_{C}$ is used for the continuous sequence-to-sequence steering torque prediction task.

$$
\operatorname{loss}_{c}=\sum_{i=1}^{L} \frac{\left(t_{i}-y_{i}\right)^{2}}{L}
$$

where $L$ is the sequence length, $t_{i}$ is the target output at step $i$, and $y_{i}$ is the model predicted output. Once the continuous steering torque prediction network is trained, the base temporal pattern extraction layer will be used for the construction of the discrete intention prediction network using the transfer learning approach. The discrete intention prediction network can be represented as follows.

$$
I_{t}=\operatorname{softmax}_{d}\left(f c_{d}\left(\text { bilstm }_{d}\left(\text { bilstm }_{c}\left(\operatorname{In}_{t}\right)\right)\right)\right)
$$

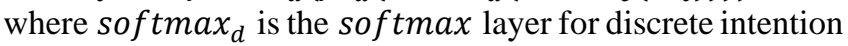
classification, $f c_{d}$ is the fully-connected layer with 100 neurons, bilstm ${ }_{d}$ is the personalized Bi-directional LSTM RNN network with 40 LSTM cells in each direction for the discrete intention prediction, and bilstm $_{c}$ is the common Bidirection LSTM RNN network as trained in the continuous intention prediction case. The input for the discrete intention classification mode $I n_{-} d_{t}$ has the same format as $I n_{-} c_{t}$. In the discrete steering intention prediction case, a varied $h$ from 100 to 800 , and a varied $p$ from 100 to 1000 will also be evaluated. The model output $I_{t}$ is an intentional state, where $I_{t} \in$ $[1,2,3,4,5]$ and represent five steering states. The five states are defined based on the variation of the steering torque, as shown in Fig. 4, to characterize different steering maneuvers.

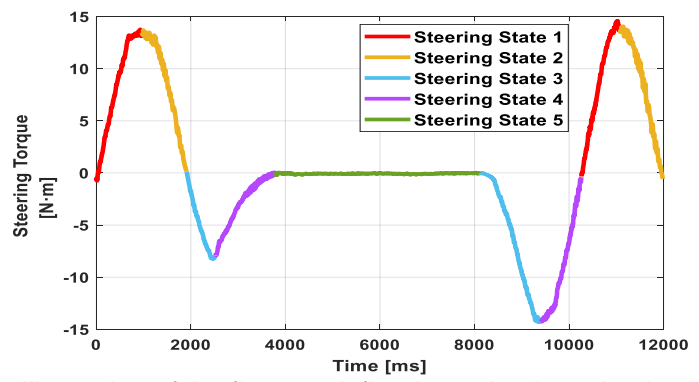

Fig. 4. Illustration of the five pre-defined steering intention based on the variation of the steering torque.
For each steering torque sequence, the steering intent is defined based on the slope of the steering torque in the final 100 $\mathrm{ms}$. To determine the state, the slope of the signal will be calculated every $10 \mathrm{~ms}$, and the sub-intent of the $10 \mathrm{~ms}$ slice is determined based on the slope and the steering torque value. Then, the final steering intent will be the majority one among the ten sub-intent.

For the discrete steering intention prediction task, a crossentropy loss function $\operatorname{loss}_{d}$ is used for the multi-class classification task, which is described as:

$$
\operatorname{loss}_{d}=-\sum_{i=1}^{N} \sum_{j=1}^{K} I_{i j} \ln y_{i j}
$$

where $N$ is the total number of samples, $K$ is the number of classes, $I_{i j}$ indicates the $\mathrm{i}^{\text {th }}$ sample belongs to the $\mathrm{j}^{\text {th }}$ class, and $y_{i j}$ is the softmax layer output indicating the probability of sample $i$ associate with the class $j$.

\section{B. Model Training and Implementation}

In this study, the sequential data are split based on the 200 ms prediction horizon and the historical horizon. In total, 25333 driving sequences from the both-hand driving mode and 26750 sequences from the single-hand driving mode are collected from the 21 participants. The training and testing data are randomly selected from the dataset, with data from $80 \%$ are used for model training and the rest is used for model testing. The construction of the time-series model follows two stages. First, the sequence-to-sequence continuous steering torque prediction model is trained. Then, the base Bi-LSTM layer of the model will be concatenated with another Bi-LSTM layer and FC layers for discrete steering intention prediction based on the transfer learning method.

The models are optimized with Adam optimizer [38]. The initial learning rate is 0.001 for the continuous intention prediction model. The max epoch is 60 , and the mini-batch size is 32. Regarding the discrete intention prediction model, the first Bi-LSTM layer of the joint model is adopted with an initial learning rate of 0.0001 to slow down the learning in the transfer layer, while the weighted learning rate of the FC layer is 10 to speed up the learning process of the new layers. The discrete intention prediction model is easier to converge than the continuous intention prediction model. Hence, the max epoch is selected as 30 for this classification network. The whole network is developed with MATLAB 2020a.

\section{EXPERIMENT RESULTS}

In this section, the model performance on the continuous and discrete steering intention prediction is evaluated. The hybridlearning-based deep time-series model is compared with several baseline methods with different evaluation metrics, and the model performances are visualized and quantitatively analyzed.

\section{A. Evaluation Metrics and Baselines}

The root-mean-square error (RMSE) is a common metric for regression evaluation $[39,40]$. It will be used in this study to evaluate the model performance on the continuous and sequential steering torque prediction. The RMSE is calculated as follows.

$$
R M S E=\frac{1}{N} \sum_{j=1,2, \cdots N} \sqrt{\frac{1}{L} \sum_{i=1,2, \cdots L}\left(\left(\hat{x}_{j i}-x_{j i}\right)^{2}\right)}
$$


where $N$ is the total number of testing sequences, $L$ is the sequence length, which is 200 in this part. $\hat{x}_{j i}$ is the $\mathrm{i}^{\text {th }}$ predicted value within the sequence $j$, and $x_{j i}$ is the corresponding ground truth value.

To evaluate the discrete intention prediction model, four metrics, namely, precision, recall, F1 score, and average precision, are adopted to investigate the model classification accuracy [41]. The Precision $\left(P_{r}\right)$ can be described as:

$$
\operatorname{Pr}=\frac{T_{p}}{T_{p}+F_{p}}
$$

The Recall $\left(R_{e}\right)$ is calculated as:

$$
\operatorname{Re}=\frac{T_{p}}{T_{p}+F_{n}}
$$

where $T_{p}$ is the true positive value, $F_{p}$ is the false positive, and $F_{n}$ is the false negative value. The F1-score considers both the $P_{r}$ and $R_{e}$, and it is the harmonic mean of these two values.

$$
F 1=2 \times \frac{P r \times R e}{P r+R e}
$$

Last, the general average precision is calculated as:

$$
G_{\text {Ave }}=\frac{\text { Total number of correct prediction }}{\text { Total number of samples }}
$$

Several baselines are used to make a comparison between the existing algorithms and the proposed method. The baselines include:

1. Feedforward Neural Network (FFNN). The FFNN passes the input vectors layer-by-layer without any feedback connections. The FFNN is a common approach for solving complex nonlinear mapping problems. An FFNN model with 30 neurons is trained to predict the steering torque at the next time step.

2. Time Delay Neural Network (TDNN). The time-delay neural network for sequence prediction is used. It enables the prediction of future torque based on short-term historical data. The two-steps time delay is selected, which allows the TDNN to predict the future steering torque based on the input states in the current step and the past two-time steps.

3. LSTM Model (LSTM). Two dual-layer LSTM models are trained for the continuous and discrete intention prediction, respectively. The LSTM model enables the sequence prediction based on historical sequential data. The LSTM model has a similar structure to the model shown in Fig. 1, while the Bi-LSTM layers are replaced with LSTM layers.

4. Bi-LSTM Model (Bi-LSTM). A dual-layer Bi-LSTM model is trained for discrete steering intention classification to evaluate the effectiveness of the hybridlearning-based model.

5. Hybrid-learning-based model (HL-BLSTM). The hybrid-learning-based continuous and discrete steering intention prediction model, as shown in Fig. 1, is developed as the proposed method.

6. Hybrid-learning-based model with Feature Selection (HL-BLSTM-F). The HL-BLSTM model is trained with selected features regarding the correlation coefficient given in Table. 1. Only the strong correlated muscle signals are used for model training. Hence, for the both-arm driving mode, EMG signals of MB2, MB6, and MB7 muscles are used. For the single-arm driving mode, the EMG signals of MB1, MB2, MB5, MB9, MB10 muscles are adopted.

\section{B. Continuous Steering Torque Prediction}

In this part, the continuous steering torque prediction results from the proposed network and baseline algorithms are evaluated and compared. The comparison of the results is shown in Table 2 below. Based on table 2, it can be found that the proposed Bi-directional LSTM based model achieved the most accurate prediction results with the both-hand and singlehand driving mode. The HL-BLSTM achieved $0.5820 \mathrm{~N} \cdot \mathrm{m}$ RMSE in the both-hand driving mode and $0.6920 \mathrm{~N} \cdot \mathrm{m}$ RMSE in the single-hand driving mode. The LSTM model has the same structure as the proposed network, while the precision is much lower than the HL-BLSTM mode, which means the Bidirectional framework is a more effective structure. Another conclusion that can be made in Table 2 is that the prediction accuracy for the both-hand driving mode is uniformly higher than that in the single-hand driving case with different algorithms. This shows that with the both-hand driving mode and the ten EMG signals, the driver steering behavior can be easier to be predicted than that with the single-hand driving mode. Regarding the HL-BLSTM-F models of the both-hand and single-hand driving modes, comparable results (with slightly higher RMSE) are achieved compared to the models that are trained with full feature sets.

TABLE II

RESULTS COMPARISON OF THE CONTINUOUS STEERING TORQUE PREDICTION

\begin{tabular}{ccc}
\hline \hline Algorithms & $\begin{array}{c}\text { Both-Hand RMSE }[N \cdot \\
m]\end{array}$ & Single-Hand RMSE $[N \cdot m]$ \\
\hline FFNN & 2.5580 & 2.6800 \\
TDNN & 8.9020 & 9.6060 \\
LSTM & 1.5480 & 1.9200 \\
HL-BLSTM & 0.5820 & 0.6920 \\
HL-BLSTM- & 0.6239 & 0.7170 \\
F & & \\
\hline \hline
\end{tabular}

The performance visualization of the proposed method is shown in Fig. 5. It is shown that the proposed network can efficiently predict the steering torque with the $200 \mathrm{~ms}$ prediction and historical horizon. While it is also clear to see that the largest prediction error usually happens at the points where maximum or the minimum steering torque occurs. This phenomenon could be caused by the nature of the regression model and the loss function, which is to find the minimum overall regression error as the number of data points at the peak value of the sine-wave-like signal is smaller than that at the constant variation and steady states. Also, the regression model tends to step back to be more stable for maintaining accurate 

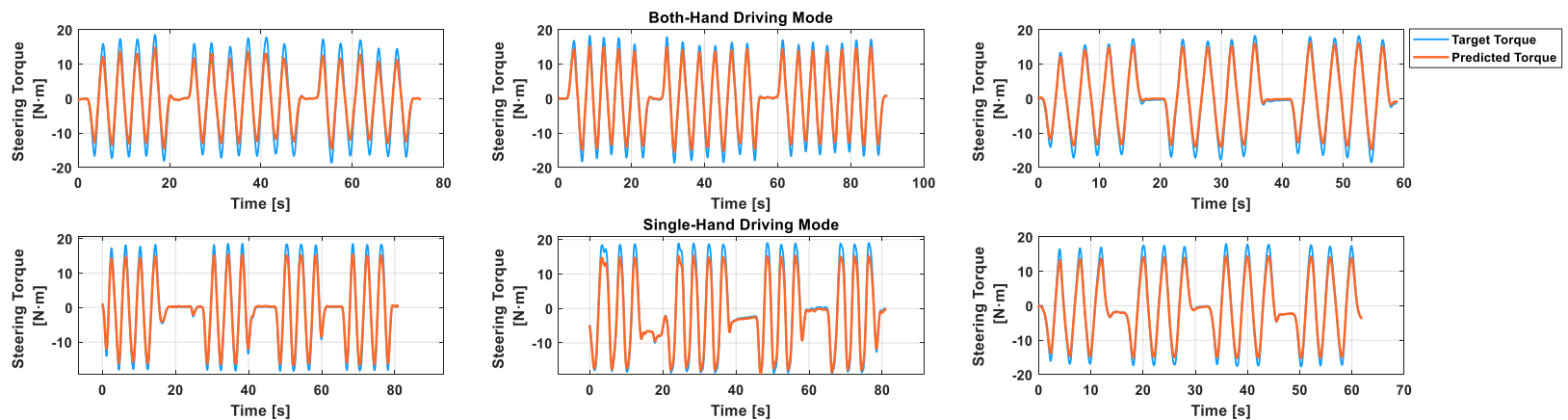

Fig.5. Visualization of the continuous steering torque prediction results with the Bi-directional LSTM model on different testing scenarios. The upper row indicates the prediction results for both-hand driving mode, and the bottom row shows the results for three single-hand driving mode cases. prediction at the steady states. Hence, the model generates a larger prediction error at the peak points to ensure small global average prediction errors. In general, the model can accurately predict the steering torque during the steering maneuver and the steady stage.

\section{Discrete Steering Intention Prediction}

In this part, the discrete steering intention prediction results of the proposed model are evaluated from three aspects, which are performance comparison with baseline methods, accuracy analysis among different intent, and the evaluation of the impact of prediction and historical horizon on the model classification accuracy.

First, the model comparison based on different algorithms is shown in Table 3 and Table 4 for both-hand driving mode and the single-hand driving mode, respectively. As shown in Table 3 and Table 4, the proposed HL-BLSTM model achieved the most accurate classification results for the five steering intents with the two different driving modes. Specifically, the proposed HL-BLSTM model generates $95.34 \%$ and $94.40 \%$ mean accuracy in the intention prediction task, respectively. Similar to the results in the continuous steering torque prediction case, the both-hand driving mode always leads to a higher steering intention classification accuracy than that with the single-hand driving mode.

Also, it is found that the proposed HL-BLSTM model, which is trained with the transfer learning method, can achieve more accurate classification results than the Bi-LSTM model, which is trained from scratch. Also, the Bi-directional LSTM structure shows a significant advantage to the conventional LSTM model and feedforward neural network structure. Considering the impact of feature selection on the model performance, similar to the continuous steering torque prediction, the HL-BLSTM-F achieved comparable prediction accuracy as the HL-BLSTM on the discrete steering intention classification. The general average precision for the HL-BLSTM-F model in the both-hand and single-hand driving mode is $94.99 \%$ and $92.97 \%$, respectively. This shows that the driver steering intent can be accurately predicted with very limited EMG features, which can further help improve the effectiveness of the method in a realworld application.

TABLE III

RESULTS COMPARISON OF THE DISCRETE STEERING INTENTION PREDICTION WITH BOTH-HAND DRIVING MODE

\begin{tabular}{lcccc}
\hline \hline Algorithms & $\operatorname{Pr}$ & $\operatorname{Re}$ & $F 1$ & $G_{\text {Ave }}$ \\
\hline FFNN & 0.7735 & 0.7611 & 0.7634 & 0.7762 \\
LSTM & 0.8705 & 0.8717 & 0.8699 & 0.8780
\end{tabular}

\begin{tabular}{lllll} 
Bi-LSTM & 0.9406 & 0.9405 & 0.9405 & 0.9420 \\
HL-BLSTM & 0.9524 & 0.9508 & 0.9523 & 0.9534 \\
HL-BLSTM- & 0.9502 & 0.9473 & 0.9487 & 0.9499 \\
F & & & & \\
\hline \hline
\end{tabular}

TABLE IV

RESULTS COMPARISON OF THE DISCRETE STEERING INTENTION PREDICTION WITH SINGLE-HAND DRIVING MODE

\begin{tabular}{lcccc}
\hline \hline Algorithms & $\operatorname{Pr}$ & $\operatorname{Re}$ & $F 1$ & $G_{\text {Ave }}$ \\
\hline FFNN & 0.7447 & 0.7073 & 0.7129 & 0.7441 \\
LSTM & 0.8499 & 0.8041 & 0.8096 & 0.8455 \\
Bi-LSTM & 0.9372 & 0.9282 & 0.9323 & 0.9360 \\
HL-BLSTM & 0.9425 & 0.9370 & 0.9360 & 0.9440 \\
HL-BLSTM- & 0.9307 & 0.9236 & 0.9264 & 0.9297 \\
F & & & & \\
\hline \hline
\end{tabular}

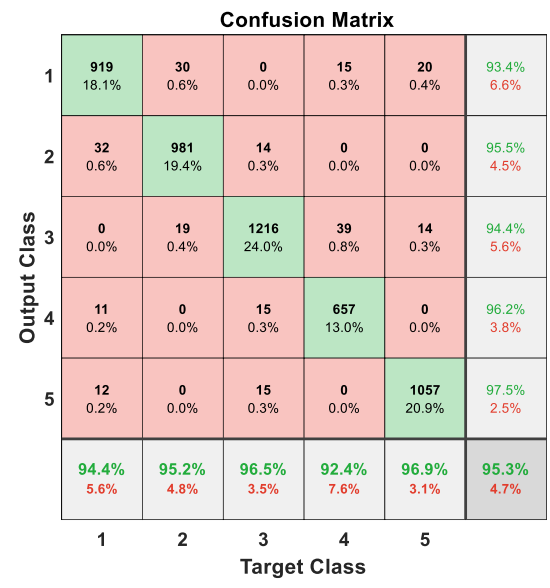

Fig. 6. Confusion matrix for the five steering intention classification with the both-hand driving mode.

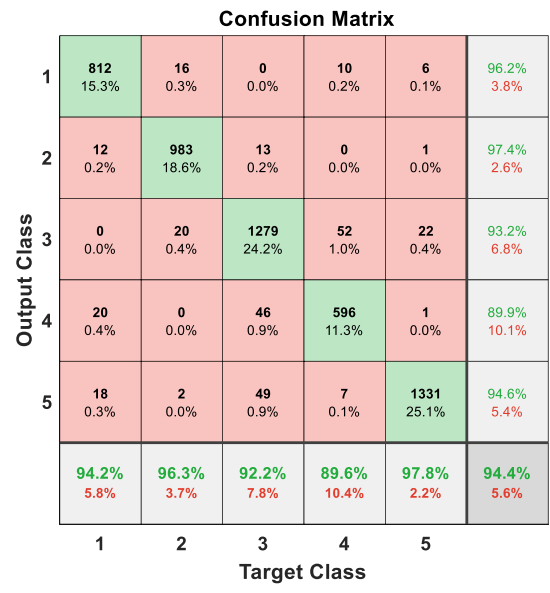


Fig. 7. Confusion matrix for the five steering intention classification with the single-hand driving mode.
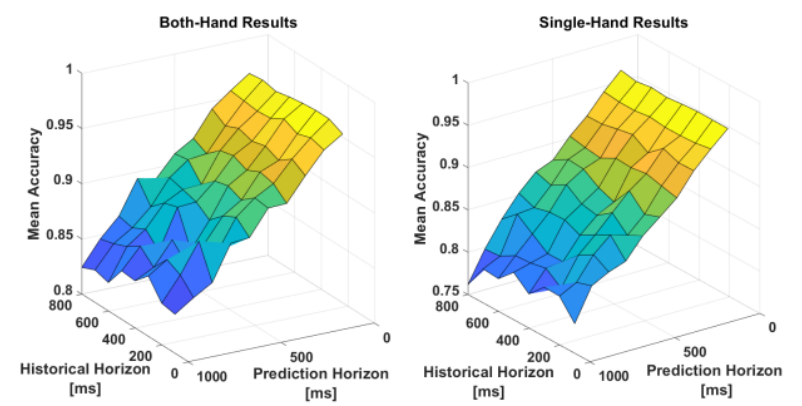

Fig. 8. Evaluation of the impact of the prediction and historical horizon on the intention classification accuracy. The bright yellow indicates a higher accuracy, while the dark blue shows a lower prediction accuracy for steering intention classification.

The HB-BLSTM model classification results on the five steering intents with both-hand and single-hand driving modes are shown in Fig. 6 and Fig. 7. In Fig. 6 and Fig. 7, the bottom rows show the classification accuracy of each class with respect to the ground truth label, and the rightmost columns show the classification results with respect to the prediction value. The green boxes show the accurate classification cases with respect to the ground truth, while the red boxes show the misclassification cases. The both-hand and single-hand driving modes generate a $95.3 \%$ and $94.4 \%$ mean classification result, respectively. The proposed model can achieve high prediction accuracy with the two driving modes. For the two driving modes, the steady steer wheel holding intent (steering state 5) achieved the most accurate prediction results $(96.9 \%$ and $97.8 \%$, respectively). While the classification results for the steering state 4 are the lowest for both of the two driving modes (92.4\% and $89.6 \%)$. It is shown that the model is more likely to misclassify the steering state 4 into the state 3 group than the other groups.

The quantitative analysis of the impact of the prediction horizon and the historical horizon on the intention classification are shown in Fig. 8. The prediction horizon is ranged from 100 $\mathrm{ms}$ to $1000 \mathrm{~ms}$, and the historical horizon is varied from $100 \mathrm{~ms}$ to $800 \mathrm{~ms}$, respectively. The prediction horizon determines how far the model can predict, and the historical horizon controls how much input data can be used. To evaluate the model performance, each model is trained three times with the specific input and output data, and the accuracy is the mean value of the three classification results. As shown in Fig. 8, the steering intention classification performance for the both-hand driving mode is always higher than $80 \%$, which is generally more accurate than that for the single-hand driving mode. Moreover, compared to the historical horizon, the prediction horizon has a significantly larger impact on model performance. The model classification accuracy on the steering intention will not increase dramatically by increasing more history input data. However, the model performance can decrease if a larger prediction horizon is chosen since the future steering intention can be highly random, and it unreasonable to infer the future intent with a very long prediction horizon.

\section{Discussions and Future Works}

In this study, a hybrid-learning-based driving steering intention prediction system is proposed for joint estimation of continuous steering torque and discrete steering intention prediction. The proposed method shows its advantage in the two tasks. Regarding the continuous steering torque prediction with Bi-LSTM, the bi-directional LSTM cell can capture the feature dependency from the forward and backward direction, which leads to higher feature representation ability compared to the conventional LSTM-based approach. Also, the Bi-LSTM model has a deeper architecture than the conventional multilayer perceptron neural networks, which carries more sequential features and contributes to a more accurate prediction of future steering torques. For the discrete steering intention prediction, the proposed hybrid learning-based approach shows higher prediction accuracy than the baseline methods.

Based on the performance analysis and evaluation from different algorithms, it is found that the both-hand driving mode always leads to higher prediction accuracy for the continuous and discrete steering intention prediction tasks than the singlehand driving mode. The reasons can be multifold. For example, the both-hand driving mode has different driving postures compared to the single-hand driving mode, which may influence the neuromuscular dynamics and makes the steering intention easier to be predicted. Moreover, different EMG signals are measured for the two driving modes, which can also lead to different prediction results. However, it is clear that the ten EMG signals from the both-hand driving mode can be more efficient features than those from the single-hand driving mode for driver behavior understanding and intention prediction. This result can contribute to a better mutual-understanding enabled driver-vehicle-collaboration system.

In this study, the impact of the prediction and historical horizon on the steering intention prediction is also evaluated. Regarding the time delay analysis, it is shown that most of the EMG signals show a 100 to $200 \mathrm{~ms}$ time lag to the steering torque. Hence, a $200 \mathrm{~ms}$ history and prediction horizon is reasonable for the steering intention prediction. It is also found that increasing the historical horizon and involve more historical data will not improve the prediction accuracy but increase the system memory and computational burden. Meanwhile, the model performance will decrease if a broader prediction horizon is selected. As the future steering intention can be influence by many aspects, such as the traffic context and driver tactical intention, a longer-term driver intention prediction (several seconds ahead) should not only rely on the neuromuscular dynamics and historical steering behaviors.

The sine-wave like steering task and the five steering intention classifications represent typical daily driving behaviors. The reason for choosing such driving scenarios is multifold. First, based on the prediction horizon evaluation, it can be found that a reasonable estimation for the steering torque and intention is about $200 \mathrm{~ms}$, which means the neuromuscular dynamics are more suitable for short-term steering intention prediction. Increasing the complexity of driving scenarios will have a limited influence on the prediction capability of the EMG-based intent prediction system. However, it is also important to analyze the neuromuscular dynamics during the 
critical steering situation to gain a comprehensive understanding of the EMG-based steering intention prediction system. Second, based on current shared control and take-over control studies, the most critical movement for driver-vehicle interaction is the transition period from automation to manual control. It is important to know the drivers' steering intention after they take-over the control authority [42,43]. Hence, the proposed system will benefit the design of a future drivervehicle interaction system for an automated driving vehicle as both continuous and discrete steering intention can be predicted.

It should be noted that the overall EMG sensor setup in this study could be time-consuming for real-world applications. However, several potential solutions can be expected to improve the acceptance and generalization of the proposed system. First, it is found that using EMG signals that are highly correlated to the steering torque can achieve compatible accuracy with much fewer sensors. Second, the EMG-based system can be popularized with the development of wearable sensors so that the acceptance and usage of such a system can be improved [44]. Furthermore, the proposed system has great potential in specific application domains where assistive machines are needed, for example, assistive mobility devices for elder or disabled people.

Based on the proposed driver steering intention prediction system, future works can concentrate on the development of an advanced intention inference system based on a holistic signal processing and sensor fusion system to achieve longer-term intention and steering behavior prediction. Moreover, the impact of different driving postures and hand positions on the driver intention understanding can be evaluated further. It was shown that different driving postures do influence the sequential steering torque prediction for the both-hand driving mode, with the 3 -clock driving posture contributes to the most accurate prediction results [45]. However, how the driving postures influence steering intention with different prediction horizon and driving mode need to be further investigated. It is also interesting to use feature engineering knowledge to select the most critical EMG features and reduce the dimension of model input. Last, human-centered shared control and take-over control system for the automated driving vehicle can be developed by integrating the driver intent prediction system to exploit how mutual understanding can benefit collaborative automated driving.

\section{CONCLUSION}

In this study, a hybrid-learning-based driver steering intention system using the neuromuscular dynamics is proposed. The system is developed to predict both the continuous driver steering torque as well as the discrete steering direction based on the deep Bi-LSTM-RNN model. Based on the time delay analysis, the impacts of the historical horizon, and the prediction horizon on the steering intention prediction are investigated. It is found that the model can achieve a precise steering torque prediction with a $200 \mathrm{~ms}$ prediction horizon. The prediction RMSEs for the single-hand and both-hand are $0.692 N \cdot m$ and $0.582 N \cdot m$, respectively. Meanwhile, the model can accurately predict the discrete steering intention with an even larger prediction horizon. Also, it is shown that the prediction horizon is more important than the historical horizon in the steering intention prediction. The proposed system can be an efficient and critical component for the shared control and take-over performance assessment system for automated driving vehicles.

\section{REFERENCES}

[1] Chen, Jessie YC, and Michael J. Barnes. "Human-agent teaming for multirobot control: A review of human factors issues." IEEE Transactions on Human-Machine Systems 44.1 (2014): 13-29.

[2] Nguyen, Anh-Tu, Chouki Sentouh, and Jean-Christophe Popieul. "Driverautomation cooperative approach for shared steering control under multiple system constraints: Design and experiments." IEEE Transactions on Industrial Electronics 64.5 (2016): 3819-3830.

[3] Xing, Yang, et al. "Driver lane change intention inference for intelligent vehicles: framework, survey, and challenges." IEEE Transactions on Vehicular Technology 68.5 (2019): 4377-4390.

[4] Lv, Chen, et al. "Characterization of driver neuromuscular dynamics for human-automation collaboration design of automated vehicles." IEEE/ASME Transactions on Mechatronics 23.6 (2018): 25582567.

[5] Shin, Donghoon, et al. "Human-centered risk assessment of an automated vehicle using vehicular wireless communication." IEEE Transactions on Intelligent Transportation Systems 20.2 (2018): 667-681.

[6] SAE International. Taxonomy and Definitions for Terms Related to Driving Automation Systems for On Road Motor Vehicles. 2016.

[7] Beiker, Sven A. "Legal aspects of autonomous driving." Santa Clara L. Rev. 52 (2012): 1145.

[8] P. M. Fitts, Human Engineering for an Effective Air Navigation and Traffic Control System. Washington, DC: National Research Council, 1951.

[9] Christoffersen, Klaus, and David D. Woods. "How to make automated systems team players." Advances in human performance and cognitive engineering research 2 (2002): 1-12.

[10] Endsley, Mica R. "From here to autonomy: lessons learned from humanautomation research." Human factors 59.1 (2017): 5-27.

[11] Ji, Xuewu, et al. "Shared steering torque control for lane change assistance: a stochastic game-theoretic approach." IEEE Transactions on Industrial Electronics 66.4 (2018): 3093-3105.

[12] Zhang, Bangji, et al. "A novel observer design for simultaneous estimation of vehicle steering angle and sideslip angle." IEEE Transactions on Industrial Electronics 63.7 (2016): 4357-4366.

[13] Benloucif, Amir, et al. "Cooperative trajectory planning for haptic shared control between driver and automation in highway driving." IEEE Transactions on Industrial Electronics 66.12 (2019): 9846-9857.

[14] Flemisch, Frank, et al. "Shared control is the sharp end of cooperation: Towards a common framework of joint action, shared control and human machine cooperation." IFAC-PapersOnLine 49.19 (2016): 72-77.

[15] Guo, Hongyan, et al. "Hazard-evaluation-oriented moving horizon parallel steering control for driver-automation collaboration during automated driving." IEEE/CAA Journal of Automatica Sinica 5.6 (2018): 1062-1073.

[16] Zeeb, Kathrin, Axel Buchner, and Michael Schrauf. "Is take-over time all that matters? The impact of visual-cognitive load on driver take-over quality after conditionally automated driving." Accident Analysis \& Prevention 92 (2016): 230-239.

[17] Bazilinskyy, Pavlo, et al. "Take-over requests in highly automated driving: A crowdsourcing survey on auditory, vibrotactile, and visual displays." Transportation research part F: traffic psychology and behaviour 56 (2018): 82-98.

[18] Xie, Guotao, et al. "Vehicle trajectory prediction by integrating physicsand maneuver-based approaches using interactive multiple models." IEEE Transactions on Industrial Electronics 65.7 (2017): 59996008.

[19] Y. Xing, and C. Lv. "Dynamic State Estimation for the Advanced Brake System of Electric Vehicles by using Deep Recurrent Neural Networks." IEEE Transactions on Industrial Electronics (2019).

[20] Li, Xiaohan, Wenshuo Wang, and Matthias Roetting. "Estimating driver's lane-change intent considering driving style and contextual traffic." IEEE 
Transactions on Intelligent Transportation Systems 20.9 (2018): 32583271.

[21] Jain, Ashesh, et al. "Car that knows before you do: Anticipating maneuvers via learning temporal driving models." Proceedings of the IEEE International Conference on Computer Vision. 2015.

[22] Xing, Yang, et al. "An ensemble deep learning approach for driver lane change intention inference." Transportation Research Part C: Emerging Technologies 115 (2020): 102615.

[23] Jain, Ashesh, et al. "Brain4cars: Car that knows before you do via sensoryfusion deep learning architecture." arXiv preprint arXiv:1601.00740 (2016).

[24] Doshi, Anup, and Mohan Manubhai Trivedi. "On the roles of eye gaze and head dynamics in predicting driver's intent to change lanes." IEEE Transactions on Intelligent Transportation Systems 10.3 (2009): 453-462.

[25] Jang, Giho, et al. "EMG-based continuous control scheme with simple classifier for electric-powered wheelchair." IEEE Transactions on Industrial Electronics 63.6 (2016): 3695-3705.

[26] Bi, Luzheng, et al. "Development of a driver lateral control model by integrating neuromuscular dynamics into the queuing network-based driver model." IEEE Transactions on Intelligent Transportation Systems 16.5 (2015): 2479-2486.

[27] Ding, Qichuan, et al. "Missing-data classification with the extended fulldimensional Gaussian mixture model: Applications to EMG-based motion recognition." IEEE Transactions on Industrial Electronics 62.8 (2015): 4994-5005.

[28] Abbink, David A., et al. "The importance of including knowledge of neuromuscular behaviour in haptic shared control." 2012 IEEE International Conference on Systems, Man, and Cybernetics (SMC). IEEE, 2012.

[29] Liu, Jun, et al. "Driver-automation shared steering control for highly automated vehicles." Science China Information Sciences 63.9 (2020): 116.

[30] Pick, Andrew J., and David J. Cole. "Measurement of driver steering torque using electromyography."Journal of Dynamic Systems, Measurement, and Control. (2006): 960-968.

[31] Hayama, Ryouhei, et al. "Preliminary research on muscle activity in driver's steering maneuver for driver's assistance system evaluation." Proceedings of the FISITA 2012 World Automotive Congress. Springer, Berlin, Heidelberg, 2013.

[32] Liu, Yahui, et al. "A study on objective evaluation of vehicle steering comfort based on driver's electromyogram and movement trajectory." IEEE Transactions on Human-Machine Systems 48.1 (2017): 41-49.

[33] Liu, Yahui, et al. "Method for measuring a driver's steering efficiency using electromyography." Proceedings of the Institution of Mechanical Engineers, Part D: Journal of Automobile Engineering 228.10 (2014): 1170-1184.

[34] Xing, Yang, et al. "Pattern Recognition and Characterization of Upper Limb Neuromuscular Dynamics during Driver-Vehicle Interactions." Iscience 23.9 (2020): 101541.

[35] Buck, John R., Michael M. Daniel, and Andrew Singer. Computer explorations in signals and systems using MATLAB. Upper Saddle River, NJ, USA: Prentice Hall, 2002.

[36] Schuster, Mike, and Kuldip K. Paliwal. "Bidirectional recurrent neural networks." IEEE transactions on Signal Processing 45.11 (1997): 26732681.

[37] Hochreiter, Sepp, and Jürgen Schmidhuber. "Long short-term memory." Neural computation 9.8 (1997): 1735-1780

[38] Kingma, Diederik P., and Jimmy Ba. "Adam: A method for stochastic optimization." arXiv preprint arXiv:1412.6980 (2014)

[39] Xing, Yang, et al. "Energy oriented driving behavior analysis and personalized prediction of vehicle states with joint time series modeling." Applied Energy 261 (2020): 114471.

[40] Alahi, Alexandre, et al. "Social 1stm: Human trajectory prediction in crowded spaces." Proceedings of the IEEE conference on computer vision and pattern recognition. 2016.

[41] Hanley, James A., and Barbara J. McNeil. "The meaning and use of the area under a receiver operating characteristic (ROC) curve." Radiology 143.1 (1982): 29-36.

[42] Li, Mingjun, et al. "Shared control driver assistance system based on driving intention and situation assessment." IEEE Transactions on Industrial Informatics 14.11 (2018): 4982-4994.

[43] Wintersberger, Philipp, et al. "Let me finish before I take over: Towards attention aware device integration in highly automated vehicles." Proceedings of the 10th International Conference on Automotive User Interfaces and Interactive Vehicular Applications. 2018.

[44] Doheny, Emer P., et al. "Feature-Based Evaluation of a Wearable Surface EMG Sensor Against Laboratory Standard EMG During Force-Varying and Fatiguing Contractions." IEEE Sensors Journal 20.5 (2019): 27572765.

[45] Xing, Yang, et al. "Continuous Driver Steering Intention Prediction Considering Neuromuscular Dynamics and Driving Postures." 2020 IEEE International Conference on Systems, Man, and Cybernetics (SMC). IEEE, 2020

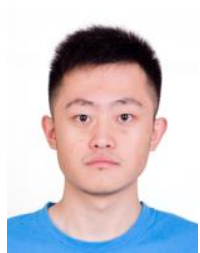

Yang Xing received his Ph.D. degree from Cranfield University, UK, in 2018. He is currently a Research Associate with the Department of Computer Science, University of Oxford, UK. He was a Research Fellow with the School Mechanical and Aerospace Engineering, Nanyang Technological University, Singapore. His research interests include machine learning, human behavior modeling, intelligent multi-agent collaboration, and intelligent/autonomous vehicles. Dr. Xing serves as a Guest Editor for IEEE Internet of Thing, IEEE Intelligent Transportation Systems Magazine, and Frontiers in Mechanical Engineering.

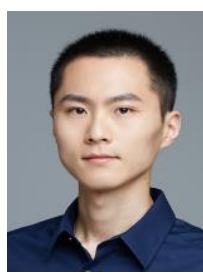

Chen $\mathbf{L v}$ is currently an assistant professor at Nanyang Technology University, Singapore. He received a Ph.D. degree at the Department of Automotive Engineering, Tsinghua University, China in 2016. From 2014 to 2015, he was a joint Ph.D. researcher at EECS Dept., University of California, Berkeley. His research focuses on advanced vehicle control, where he has contributed over 100 papers and obtained 12 granted patents.

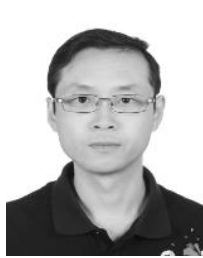

Yahui Liu received his B.S. degree from Jilin University, China, in 2003 and Ph.D. degree from Beihang University, China, in 2009 respectively. He is currently an Assistant Professor at State Key Laboratory of Automotive Safety and Energy, Tsinghua University, China. His research interests include driver-vehicle system dynamics, steering system, and actuator design of ADAS.

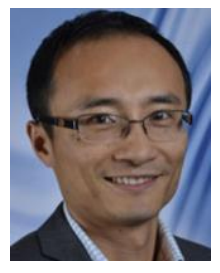

Yifan Zhao was born in Zhejiang, China. He received the Ph.D. degree in automatic control and system engineering from the University of Sheffield, Sheffield, U.K., in 2007. $\mathrm{He}$ is currently a Lecturer in image and signal processing and degradation assessment at Cranfield University, Cranfield, U.K. His research interests include computervision-based process monitoring, super resolution, active thermography, and nonlinear system identification.

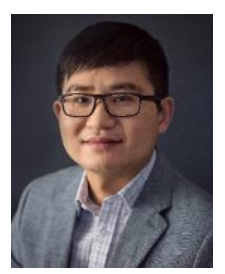

Dongpu Cao received the Ph.D. degree from Concordia University, Canada, in 2008. He is currently an Associate Professor and Director of Driver Cognition and Automated Driving (DC-Auto) Lab at the University of Waterloo, Canada. His research focuses on vehicle dynamics and control, driver cognition, automated driving, and parallel driving, where he has contributed more than 150 publications and 1 US patent.

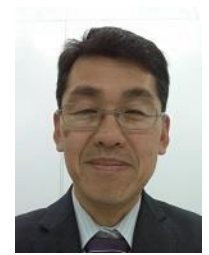

Sadahiro Kawahara had 15 years' experience as engineer, researcher and R\&D manager in R\&D center of JTEKT CORPORATION, after 13 years career as an engineer of driver control devices in NISSAN MOTOR CORPORATION. His major research topics are steering and vehicle system control including human machine interface and autonomous driving. 
2021-02-23

Hybrid-learning-based driver steering intention prediction using neuromuscular dynamics

Xing, Yang

IEEE

Xing Y, Lv C, Liu Y, et al., (2022) Hybrid-learning-based driver steering intention prediction using neuromuscular dynamics. IEEE Transactions on Industrial Electronics, Volume 69, Number 2, February 2022, pp. 1750-1761

http://doi.org/10.1109/TIE.2021.3059537

Downloaded from Cranfield Library Services E-Repository 